\title{
Visual discrimination learning of random figure problems by rhesus monkeys*
}

\author{
KRISTIN R. CARLSON $\dagger$ \\ Department of Pharmacology, University of Pittsburgh School of Medicine, Pittsburgh, Pennsylvania 15213
}

\begin{abstract}
In a two-choice discrimination learning paradigm, a U-shaped function was found between trials to criterion and the number of sides comprising the random figure discriminanda. These data are consistent with results from human Ss and suggest similar information processing capabilities in the two species. Changing the orientation of the stimulus figures after the discrimination had been learned only partially disrupted subsequent discrimination performance. Any single difference in selected form attributes between the two figures comprising a problem was not a good predictor of learning speed, suggesting that monkeys use a combination of such factors.
\end{abstract}

In the study of human form perception, random figures have been used extensively (for review see Zusne, 1970). These figures are generated by randomly assigning points to coordinates in a 100 by 100 matrix and by connecting the points with straight lines, according to a set of rules, to form closed polygons (Attneave \& Arnoult, 1956). Figures composed of 4 to 16 sides are commonly used; some examples are shown in Fig. 1.

Random figures have several advantages over other types of forms, such as alphanumeric characters, or the "junk" objects commonly used with monkeys. They are unfamiliar to human or animal Ss, and an infinitely large population can be constructed, a requisite for many investigations, e.g., learning set formation. Furthermore. their metrics are precisely specifiable; measures such as length of lines, relation of perimeter to area, and so forth, can be determined. Such specifications are a first step toward deriving the form attributes of stimulus objects which are important in discrimination learning (Michels \& Zusne, 1965).

The most basic form characteristic, the number of sides comprising the figures, has been systematically varied in several studies of human discrimination learning. From this work, an interesting U-shaped function emerges. For example, Crook (1957) found that identification of 8 -sided figures was easier than that of 4- or 16-sided forms. Furthermore, in studies of latency to discriminate between pairs of figures (Brown, Hitchcock, \& Michels, 1962) or latency to recognize a standard form in an array of figures (Owen \& Brown, 1970), a similar U-shaped function was found. Latencies

*This investigation was supported by Grant MH20121 from the National Institute of Mental Health. The technical assistance of G. Reichert, V. Trammell, and G. Yourick is gratefully acknowledged. Portions of this study were presented at the 1972 meeting of the Society for Neuroscience.

tRequests for reprints should be sent to Kristin R. Carlson, Department of Pharmacology, 620 Scaife Hall, University of Pittsburgh School of Medicine, Pittsburgh, Pennsylvania 15213. were shortest at the 8-sided level, longer at 4 sides, and progressively increased from 8 to 20 sides. These results have been interpreted as suggesting that information processing is most efficient at intermediate sidedness levels.

Considering the widespread use of random figures with human Ss and their superiority in some major respects over other types of discrimination objects, it is surprising that they have been employed in only three
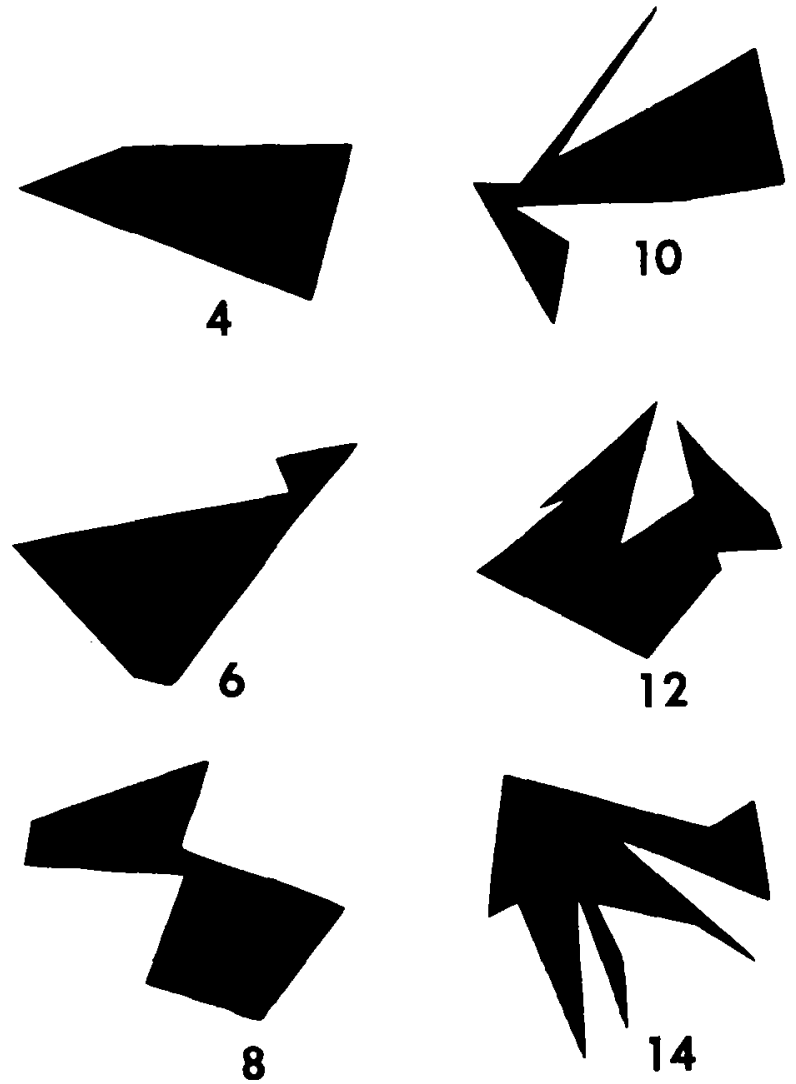

Fig. 1. Examples of the random figures used in this study. The number of sides comprising each figure is indicated beneath it. 
experiments using animal Ss. The errors made by raccoons (Fisher. 1959) and tree squirrels (Michels, Pittman. Hitchcock. \& Brown. 1962) decrease as sidedness level increases from four to seven sides. In addition. squirrel monkeys learn faster with six-sided than with four-sided figures (Nash \& Michels, 1966). These results are consistent with the studies employing humans cited earlier, in that they correspond to the first half of the U-shaped function.

Thus, the primary objective of the present study was to investigate a more extensive range of sidedness levels, using the rhesus monkey. The question posed was whether the U-shaped function between number of sides and discrimination performance generated by humans would be duplicated by nonhuman primates, thereby suggesting a basic similarity in information processing capabilities.

Of the "transpositional parameters" (Zusne, 1970), possibly the orientation of stimulus objects has been most investigated. The transfer of responses to the same form. rotated into a different orientation, has been evaluated in many species (for review see Sutherland, 1961). It is clear that both humans and monkeys can recognize a rotated object (Hunton \& Hicks, 1965), but that for humans, the speed and accuracy of matching to sample deteriorates when test figures are rotated (Woodring \& Alluisi, 1966). Furthermore, when both figures are repeatedly rotated during the course of discrimination learning, monkeys make more errors than when the objects remain in the same orientation throughout (Hicks, 1967). None of these studies employed random figures, however, and none of them evaluated the possible disruption of performance in monkeys produced by stimulus figure rotation after a problem had been learned. Thus, the second objective of this study was to determine to what extent rotation affected the performance of random figure problems, as a test of the generalizability of phenomena found with this stimulus population.

It was noted earlier that one advantage of random figures was that their metrics could be specified. Indeed, numerous form attributes other than sidedness level have been measured, and their effects on discriminability have been assessed, primarily in human Ss. Such simple characteristics as dispersion and elongation, as well as the more elaborate factor-analyzed attributes of Owen and Andolsek (1970), have been shown to be good predictors of recognition, similarity judgments, and other indicators of discriminability (Zusne, 1970). Thus, the final objective of the present study was to analyze the contribution of selected form parameters to learning speed during training and to performance after random figure rotation.

\section{METHOD}

\section{Subjects}

Five experimentally naive mature male rhesus monkeys
(Macaca mulatta) served as Ss. They were housed individually and maintained on ad lib food and water throughout the study.

\section{Apparatus}

A Wisconsin General Test Apparatus was used. The white stimulus tray measured $24 \mathrm{in}$. wide $\times 10 \mathrm{in}$. deep, and the two foodwells were $12 \mathrm{in}$. apart and $3 \mathrm{in}$. from the edge of the tray closest to $\mathrm{S}$. Latencies were measured by a Lafayette $1 / 1,000-\mathrm{sec}$ stopclock; intertrial intervals were measured by a Lafayette timer. The opaque forward screen and one-way rear screen were manually operated, as were the timers.

The stimulus figures were random figures, derived according to the Method 1 rules of Attneave and Arnoult (1956). They were equated to a $30-\mathrm{cm}^{2}$ area, using a photographic enlarger and a planimeter to measure area, and redrawn. These figures were cut from $1 / 2$-in.-thick black Plexiglas. Figures having 4, 6, 8 , 10,12 , and 14 sides were constructed (see Fig. 1 for examples) During testing the figures were laid flat, covering the foodwells, and chained to the tray.

\section{Procedure}

The Ss were pretrained according to the method of Schrier (1965). The discriminanda used in this phase were "junk" objects, and Ss were required to solve six two-object discrimination problems before beginning training.

Training consisted of a series of successive two-object problems, four at each of the six sidedness levels. Both objects in each problem had the same number of sides and were presented in the same orientation on each training trial. The presentation order of problems was pseudorandom, with the restriction that in each half of the series there were two problems at each sidedness level. The position of the correct object was varied pseudorandomly, with an equal number of right and left positions rewarded during each daily session. The intertrial interval was $20 \mathrm{sec}$. A trial was terminated and scored as an error if $S$ did not make a choice within $30 \mathrm{sec}$, but these times were not included in the latency data.

The Ss were given 40 noncorrection trials per day, to a criterion of $36 / 40$ correct on each problem. For two Ss, after criterion performance was attained on each problem, both figures were rotated $180 \mathrm{deg}$ and an additional 20 test trials were given. The same reinforcement contingencies were maintained.

\section{RESULTS}

As shown in Fig. 2, a statistically significant U-shaped function was obtained between number of sides and trials to criterion during training (Friedman analysis of variance, $\chi^{2}=11.12, \mathrm{df}=5, \mathrm{p}<.05$ ). It is clear that problems composed of figures with 6,8 , or 10 sides were more easily solved than those consisting of figures with a greater or lesser number of sides.

The latency data (Fig. 3) indicate that across all sidedness levels, mean latency to the correct object was consistently shorter than to the incorrect object (Wilcoxon test, $\mathrm{z}=-3.48, \mathrm{p}<.001$ ). There was no relation between sidedness level and latency to the correct object, but a significant inverted U-shaped function was found with latency to the incorrect object (Friedman, $\chi^{2}=26.7$, df $=5, p<.001$ ). It appears that Ss chose the correct object at the same speed regardless of problem difficulty, but showed longer latencies on the few trials on which they were incorrect when learning easy discriminations. Thus, trials to criterion 
and latency to the incorrect object are inversely related; a Spearman rank correlation coefficient of -.425 $(p<.05)$ substantiates this relationship.

The effects on performance of a change in orientation of the figures was assessed by comparing errors in the 20 trials on which the figures were rotated with errors in the first 20 trials of that problem and the last 20 trials before rotation. As seen in Fig. 4, mean errors after rotation bore no consistent relation to sidedness level. However, across all sidedness levels, errors after rotation were intermediate between, and significantly different from, errors on both the first trials (Wilcoxon, $z=$ $-3.50, p<.001$ ) and the last trials (Wilcoxon, $z=4.80$, $p<.001$ ). Thus, some disruption of performance was apparent, but complete lack of recognition of the figures was not.

The latency data obtained from the rotation test (Fig. 5) are not as clear-cut as that from training. There were no significant differences in mean latency to either the correct or incorrect object as a function of number of sides, and across all sidedness levels, the difference between latencies to the correct and the incorrect objects was not significant. Although not shown graphically, neither were there any differences at any sidedness level between latencies on the rotation test, as opposed to the first or last 20 trials. Thus, for the monkey in this situation, latency is not a sensitive measure of perfonnance.

Finally, a post hoc analysis of form attributes which might contribute to discrimination learning and performance was conducted. Although the random figures were not selected so as to systematically control differences on any attribute, within each sidedness level there was sufficient variation to permit the following comparisons.

In regard to the U-shaped function obtained during training, it was asked whether the two figures comprising easily solved problems were relatively more dissimilar in some form characteristic than were figures in more difficult problems. Several physical measures of each figure were made, and the difference between the two figures in each problem on a given measure was computed. Each difference is associated with the mean trials to criterion necessary to solve that problem. The correlation between mean trials and differences in each physical measure was computed across all problems, by means of a Spearman rank correlation coefficient.

Dispersion is usually characterized by perimeter ${ }^{2} /$ area (Michels \& Zusne, 1965); since area was constant in the present figures, perimeter alone was used. The correlation coefficient between the difference in dispersion and mean trials was not significant (-.312). Similarly, the correlation between mean trials and the difference in elongation, or the distance between the two most separated points of the figure, was not significant $(-.254)$.

Finally, the total complexity of each figure was calculated, according to the method of Vitz and Todd

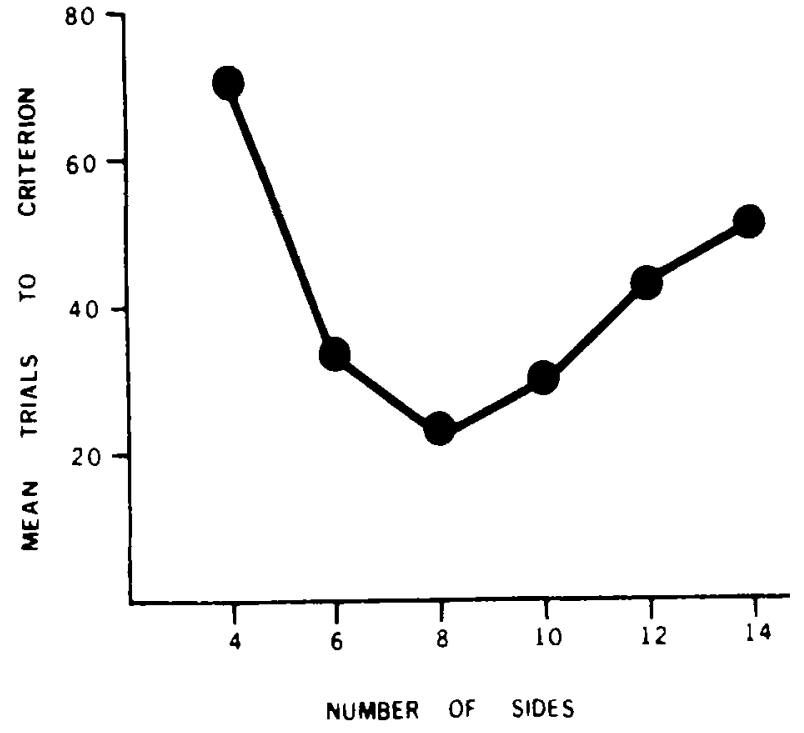

Fig. 2. Mean trials to criterion during training as a function of number of sides in the stimulus objects.

(1971). This is a computer program which essentially determines the time necessary to "see" each line and angle comprising a figure; figures with a large number of sides take longer to scan, and short sides and small angles take longer to find. As would be expected, complexity is highly correlated with sidedness level $(+.98$ for the figures used in this study). However, the correlation between the difference in complexity of the two objects

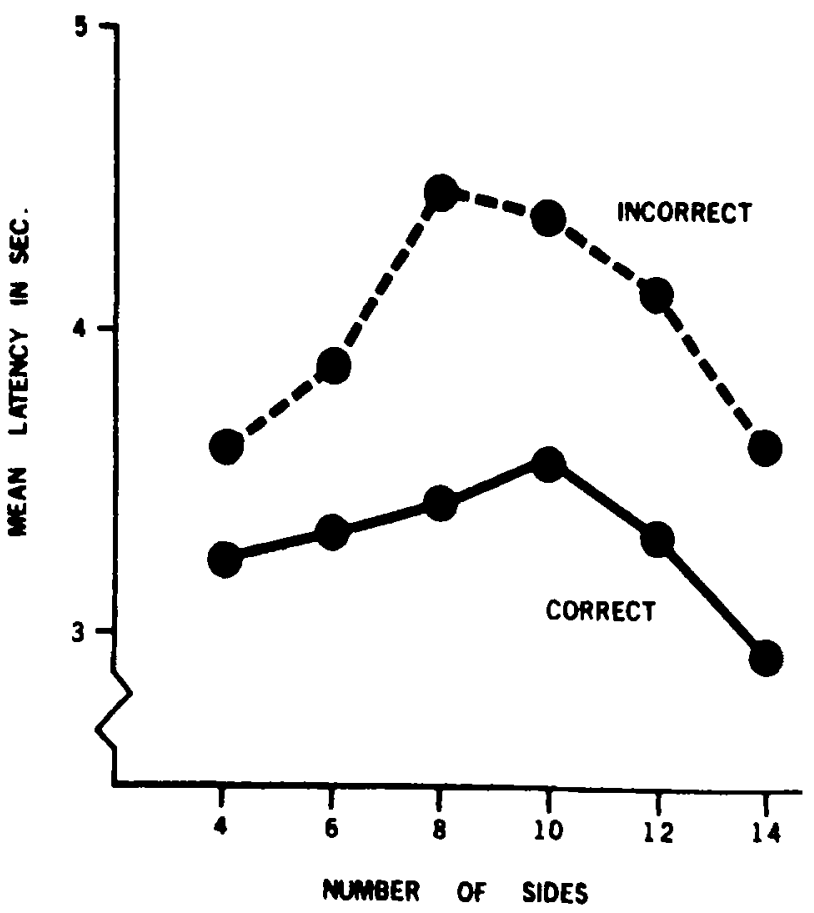

Fig. 3. Mean latency to choice during training as a function of number of sides in the stimulus objects, on trials in which the $S$ s chose the positive object (correct) and on trials in which the $S$ s chose the negative object (incorrect). 


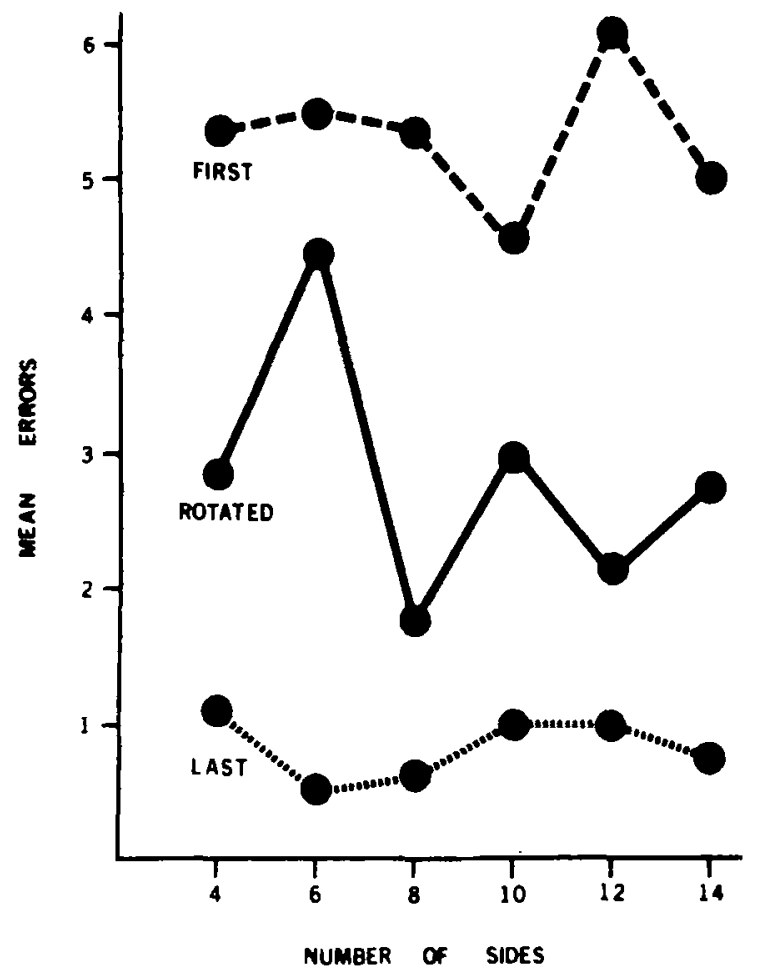

Fig. 4. Mean errors as a function of number of sides in the stimulus objects, on the first 20 trials of a problem (first), on the last 20 trials (last), and on the subsequent 20 test trials on which both objects were rotated $180 \mathrm{deg}$ (rotated).

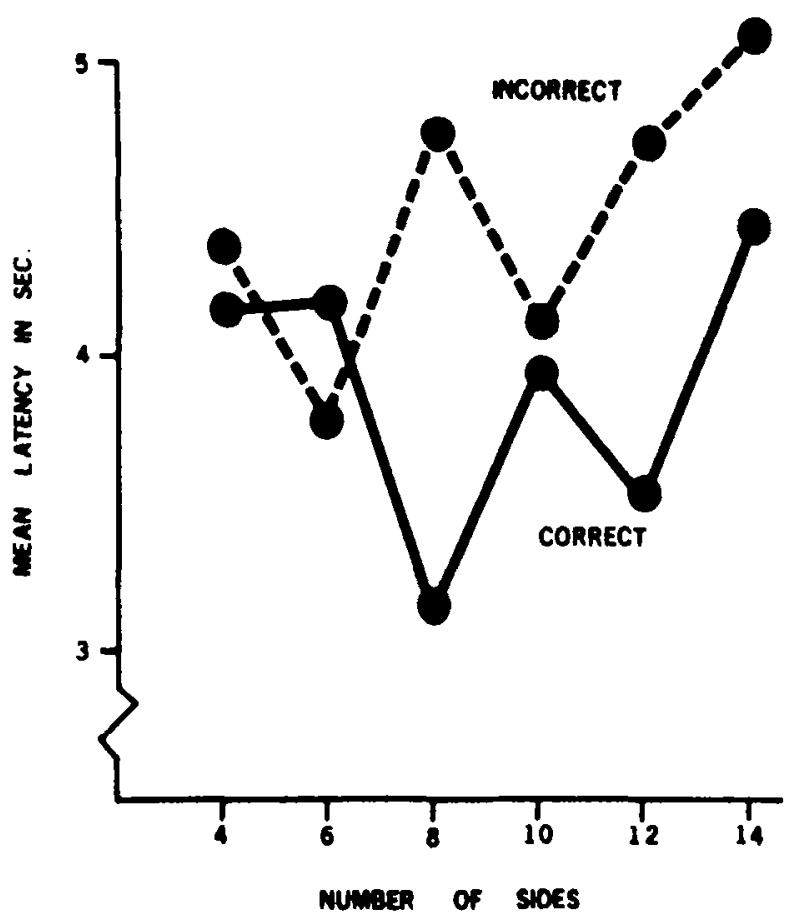

Fig. 5. Mean latency to choice during the rotation test trials as a function of number of sides in the stimulus objects, on trials in which the Ss chose the positive object (correct) and on trials in which the Ss chose the negative object (incorrect). in a problem and mean trials was not significant $(-.240)$.

Although each individual difference measure was not significantly correlated with trials to criterion, all coefficients were negative and the sum of the three difference measures was negatively and significantly correlated with trials to criterion $(-.424, p<.05)$. This indicates that some combination of these attributes was to some extent used by the Ss and that the larger the summed differences, the easier was the problem to solve.

As for correlations between these measures, either individually or summed, and errors after rotation, there were no significant relationships.

\section{DISCUSSION}

In extending the range of sidedness levels used with animals, this study demonstrates that the same U-shaped function between visual discrimination learning and sidedness level obtains with both humans and nonhuman primates, insofar as trials to criterion is concerned. It appears that for both species, not only is there an optimal level of stimulus complexity, but also this level is strikingly similar for the two species. Although the basis of this phenomenon is speculative, it is possible that at low sidedness levels, figures are not free to vary enough to be readily distinguishable, whereas figures with many sides may be too complex for efficient registration and memorization. The phylogenetic closeness of man and the rhesus monkey probably explains the observed correspondence in information handling capabilities; however, a similar study using an extended range of sidedness levels with more distant species would be valuable.

In regard to the latency data, the usual result with human Ss has been a U-shaped function, which was clearly not replicated in the present study. The discrepancy may be explained by the fact that latency to recognition in the human, particularly when under the time constraints of tachistoscopic presentation or instructions to respond promptly, is patently not the same as choice latency for a monkey in a free-response situation. The significant inverse relation between latency to the incorrect object and trials to criterion is intriguing, since it indicates that when Ss hesitate longer before choosing the negative stimulus, they learn the problem in fewer trials. Furthermore, latencies were consistently shorter to the correct than to the incorrect object, a relationship that, to the author's knowledge, has not been reported in prior investigations of primate discrimination learning. Continued attention to latency data in such studies may provide further insights into the course and determinants of discrimination performance.

The effects of stimulus figure rotation on performance were most apparent in the error data. The figures must have been partially recognized, i.e., they were not treated as comprising an entirely new problem, since errors after rotation were less than errors on the first trials. Performance was unquestionably disrupted, 
however, being significantly worse than on the last trials before rotation. These results are consistent with previous work with other types of stimulus objects, and support the generalizability of data based on this stimulus figure population.

The analyses of several form characteristics failed to reveal any single difference between stimulus objects which might account for the superior learning speed at intermediate sidedness levels or for errors after the figures were rotated. Although disappointing, this was not unexpected. The fact that the summed differences in the measures were negatively correlated with trials to criterion indicates that Ss were most likely using some combination of these and other differences, making it highly unlikely that any one measure, aside from number of sides, would be a good predictor of performance. It is possible that an extensive factor analysis, such as that used with human Ss, which employs 80 form measures factored into 10 dimensions (Owen \& Andolsek, 1970), might be useful in this regard. The numbers of Ss and stimulus figures necessary for this type of analysis are. unfortunately, prohibitively large for it to be applied to monkey discrimination learning.

A topic of considerable theoretical interest concerns the possible supramodal nature of the U-shaped relationship between stimulus complexity and efficiency of discrimination learning. Is this relation specific to information processing in the visual modality or is it illustrative of a more general sensory-nonspecific capability? Evidence from human Ss indicates that tactual errors and latencies follow the same U-shaped function (Owen \& Brown, 1970), implicating some supramodal process. Work with monkeys is presently being conducted in this laboratory to determine if tactual random figure problems are also solved most efficiently at intermediate sidedness levels.

\section{REFERENCES}

Attneave, F., \& Arnoult, M. The quantitative study of shape and pattern perception. Psychological Bulletin, 1956, 53, 452-471.
Brown, D., Hitchcock, L., \& Michels, K. Quantitative studies in form perception: An evaluation of the role of selected stimulus parameters in the visual discrimination performance of human subjests. Perceptual \& Motor Skills, 1962, 14. 519-529.

Crook, M. Facsimile-generated analogues for instrumental displays. In J. Wulfeck and J. Taylor (Eds.), Form discrimination as related to military problems. Washington. D.C: National Academy of Sciences-National Research Council, 1957. Pp. 85-98.

Fisher, B. The effects of stimulus complexity on form discrimination in Procyon lotor. Unpublished MA thesis. Purdue University, 1959. Cited by K. Michels \& L. Zusne, Metrics of visual form. Psychological Bulletin, 1965. 63. 74-86.

Hicks, L. Effects of stimulus rotation on discrimination learning by monkeys. Psychonomic Science, 1967, 9, 57-58.

Hunton, V., \& Hicks, L. Discrimination of figural orientation by monkeys and children. Perceptual \& Motor Skills, 1965. 21. 55-59.

Michels, K., Pittman, G., Hitchcock, L., \& Brown, D. Visual discrimination: Tree squirrels and quantified stimulus dimensions. Perceptual \& Motor Skills, 1962. 15, 443-450.

Michels, K., \& Zusne, L. Metrics of visual form. Psychological Bulletin, 1965, 63, 74-86.

Nash, A.. \& Michels, K. Squirrel monkeys and discrimination learning: Figural interactions, redundancies, and random shapes. Journal of Experimental Psychology, 1966, 72, 132-137.

Owen, D., \& Andolsek, M. Form recognition: Individual strategies in multivariate cue utilization. Perception \& Psychophysics, 1970, 4, 234-238.

Owen, D., \& Brown, D. Visual and tactual form discrimination: Psychophysical comparison within and between modalities. Perception \& Psychophysics, 1970, 7, 302-306.

Schrier, A. Pretraining performance of three species of macaque monkeys. Psychonomic Science. 1965, 3, 517-518.

Sutherland, N. Shape discrimination in animals. Experimental Psychology Society Monograph. No. 1. Cambridge, England: Heffner, 1961.

Vitz, P., \& Todd, T. A model of the perception of simple geometric figures. Psychological Review, 1971, 78, 207-228.

Woodring, A., \& Alluisi, E. Effects of choice-figure rotation on the visual perception of form. Psychonomic Science, 1966, 4. 403-404.

Zusne, L. Visual perception of form. New York: Academic Press, 1970.
(Received for publication August 7, 1972; accepted September 2, 1972.) 